\title{
Idosos institucionalizados: organização cronológica das rotinas diárias e qualidade do sono
}

\author{
Institutionalized elderly: chronological organization of daily routines and sleep quality \\ Ancianos institucionalizados: cronológico organización de las rutinas diarias y la calidad del sueño
}

\section{Thalyta Cristina Mansano-Schlosser', Ariene Angelini dos Santos', Simone de Oliveira Camargo-Rossignolo', Denise Cuoghi de Carvalho Veríssimo Freitas', Vera Regina Lorenz', Maria Filomena Ceolim'}

' Universidade Estadual de Campinas, Programa de Pós-Graduação em Enfermagem. Campinas-SP, Brasil.

\author{
Submissão: 30-01-2013Ａprovação: 11-06-2014
}

\section{RESUMO}

Estudo descritivo, transversal, cujo objetivo foi identificar a percepção de idosos institucionalizados quanto à organização cronológica das rotinas diárias e quanto à qualidade do sono. Participaram 37 indivíduos residentes em uma Instituição de Longa Permanência para Idosos pertencente ao município de Campinas-SP, sendo 14 mulheres e 23 homens, com média de idade de 75 anos. Verificou-se que $81 \%$ dos idosos apresentaram queixas compatíveis com sono de má qualidade, porém $70 \%$ deles informavam que tinham sono de boa qualidade, quando questionados diretamente a respeito. Todos os idosos perceberam adequadamente a organização cronológica de suas rotinas, porém essa percepção pareceu não contribuir para a boa qualidade do sono, visto que a maioria deles apresentou queixas compatíveis com sono de má qualidade. Torna-se evidente que os enfermeiros devem realizar avaliação detalhada da qualidade do sono a fim de minimizar ou prevenir esses problemas e suas possíveis consequências.

Descritores: Saúde do Idoso Institucionalizado; Instituição de Longa Permanência para Idosos; Sono; Atividades Cotidianas; Enfermagem Geriátrica.

\section{ABSTRACT}

This is a descriptive cross-sectional study, which aimed to identify the perception of nursing home elderly residents related to the chronological organization of their daily routines and to their sleep quality. The study was conducted with 37 elderly (14 women and 23 men, mean age of 75 years) who lived in a long term care facility located in the municipality of Campinas-SP, Brazil. The results showed that $81 \%$ of the elderlies had complaints compatible with poor sleep, but $70 \%$ of them reported that they had good sleep quality when directly questioned about it. All elderlies adequately realized the chronological organization of their routines, but this perception did not appear to contribute to the good sleep quality, as most of them had complaints compatible with poor sleep. It becomes evident that nurses should perform detailed assessment of sleep quality in order to minimize or prevent these problems and their possible consequences.

Key words: Health of Institutionalized Elderly; Homes for the Aged; Sleep; Activities of Daily Living; Geriatric Nursing.

\section{RESUMEN}

Estudio descriptivo, transversal, cuyo objetivo fue identificar la percepción de ancianos institucionalizados acerca de la organización cronológica de las rutinas diarias y la calidad de su sueño. Participaron en este estudio 37 ancianos (14 mujeres y 23 hombres con una edad promedia de 75 años) de un establecimiento de longa estadía para ancianos, en el municipio de Campinas-SP, Brasil. Se encontró que el $81 \%$ de los ancianos tenían quejas compatibles con sueño de mala calidad, pero el $70 \%$ de ellos informaron tener sueño de buena calidad cuando se les preguntó directamente. Los ancianos reconocían adecuadamente la organización cronológica de sus rutinas, pero esta percepción no parecía contribuir a la buena calidad del sueño, pues la mayoría tenía quejas compatibles con mala calidad. Se hace evidente que las enfermeras deben realizar una evaluación detallada de la calidad del sueño buscando minimizar o evitar estos problemas y sus posibles consecuencias.

Palabras clave: Salud del Anciano Institucionalizado; Hogares para Ancianos; Sueño, Actividades Cotidianas; Enfermería Geriátrica. 


\section{INTRODUÇÃO}

O envelhecimento da população é um fenômeno de amplitude mundial. No Brasil, foi constatado um crescimento mais acentuado do segmento populacional de 75 anos de idade ou mais, em decorrência dos avanços da medicina moderna, de melhores condições sanitárias, alimentares, ambientais e de moradia, o que acarretou em diminuição das taxas de natalidade, redução das taxas de mortalidade, resultando em aumento da expectativa de vida ${ }^{(1-2)}$. Estimativas apontam que a população idosa brasileira irá mais do que triplicar nas próximas quatro décadas. Passará de menos de 20 milhões em 2010 para aproximadamente 65 milhões em $2050^{(2)}$.

Com o envelhecimento, é possível notar mudanças na qualidade do sono dos idosos, considerada uma das queixas mais frequentes entre eles $^{(3)}$. A literatura aponta que prejuízos significativos no funcionamento cognitivo podem advir de perturbações do sono(4). Embora o processo do envelhecimento não seja considerado causa direta de problemas relacionados ao sono, algumas modificações são notadas, tais como: redução quantitativa dos estágios de sono profundo, redução do limiar para o despertar devido a ruídos, aumento quantitativo do sono superficial e da latência para o início do sono, redução da duração total do sono noturno, maior número de transições de um estágio para outro e para a vigília, maior frequência de distúrbios respiratórios durante o sono ${ }^{(5)}$. Esses fatores podem contribuir para a produção de distúrbios, como sonolência diurna excessiva e problemas cognitivos e psicológicos que levam prejuízo à qualidade de vida( ${ }^{(6)}$.

As modificações no padrão de sono e repouso têm repercussões sobre a função psicológica, sistema imunológico, desempenho, resposta comportamental, humor e habilidade de

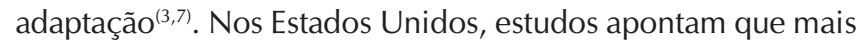
da metade dos idosos residentes na comunidade e dois terços dos institucionalizados apresentam transtornos do sono ${ }^{(8)}$.

As alterações do sono associadas ao processo de envelhecimento podem estar presentes com maior frequência ou gravidade nos residentes das Instituições de Longa Permanência para Idosos (ILPI) ${ }^{(9)}$. Sabe-se que um dos inconvenientes dessas instituições é favorecer o isolamento do idoso e sua inatividade física e mental, trazendo consequências negativas à qualidade de $v i^{(10)}{ }^{(10}$. Uma das principais alterações observadas nessas instituições é a fragmentação do sono, com despertares noturnos frequentes, que podem produzir a sonolência diurna excessiva(11).

Nas ILPIs muitas vezes não há relógios, o que prejudica a noção do tempo cronológico para o idoso. Nessa situação, é a organização das rotinas estipuladas nas ILPIs como a alimentação, a medicação, o banho, as atividades de lazer que acabam representando a marcação de tempo para os idosos. Atividades sociais, o nascer do dia e o entardecer também podem representar pistas que sinalizam o horário de acordar e o de se recolher nessas instituições ${ }^{(11)}$.

Supõe-se que os idosos que demonstram boa percepção da organização cronológica das rotinas na instituição apresentem menor número de queixas em relação ao sono, quando comparados aos idosos que não conseguem reconhecer a sequência dessas rotinas no tempo.
Diante do exposto, o conhecimento da organização cronológica das rotinas diárias e a qualidade do sono de idosos institucionalizados é essencial para subsidiar os profissionais de saúde das ILPIs no planejamento do cuidado em relação à qualidade do sono desses idosos. Nessa perspectiva, para que o enfermeiro preste uma assistência de enfermagem de qualidade torna-se necessário inteirar-se da realidade que o cerca, de modo consciente, competente, técnico e científico, sendo imprescindível a reflexão crítica para desenvolver ações a fim de prevenir, minimizar ou resolver os problemas ligados à qualidade do sono e à organização cronológica da rotina dos idosos. Pesquisas sobre essa temática são escassas em nosso contexto, havendo uma lacuna na literatura brasileira. Assim, este estudo objetivou identificar a percepção de idosos institucionalizados quanto à organização cronológica das rotinas diárias e quanto à qualidade do sono.

\section{MÉTODO}

Trata-se de um estudo descritivo, transversal, realizado em uma ILPI filantrópica, localizada em Campinas-SP. Esta ILPI possui 109 anos e abriga atualmente 150 idosos, de ambos os sexos. Os idosos independentes residem em pavilhões, separados de acordo com o sexo. Cada pavilhão é dividido em quatro quartos, nos quais dormem até nove idosos independentes. Dentro de cada pavilhão, entre os quartos, há uma pequena sala com televisor e um banheiro coletivo. Os pavilhões ocupados por indivíduos do mesmo sexo ficam próximos entre si, sendo relativamente fácil a locomoção dos idosos residentes entre estes pavilhões. $\mathrm{O}$ atendimento aos idosos é realizado por uma equipe multiprofissional, composta por equipe de enfermagem (24 horas), médico, psicólogo, dentista, nutricionista e fisioterapeuta. São realizadas periodicamente atividades de recreação e viagens turísticas com os residentes.

O projeto foi aprovado em 28/08/2007 pelo Comitê de Ética em Pesquisa (CEP) da Universidade Estadual de Campinas (Unicamp), sob o número 439/2007, CAAE 1803.0.146.000-07. Todos os cuidados éticos que regem pesquisas com seres humanos foram observados e respeitados.

Os critérios de inclusão dos sujeitos foram: residir na ILPI há no mínimo seis meses; ser independente; não possuir comprometimentos graves de linguagem ou compreensão, segundo indicação da psicóloga responsável pela instituição, confirmada pela pesquisadora por meio de uma conversa inicial; concordar em participar voluntariamente do estudo e assinar o Termo de Consentimento Livre e Esclarecido (TCLE). Os critérios de exclusão foram: encontrar-se internado na enfermaria do local durante o período de coleta de dados; ser portador de déficit visual e cognitivo que o impossibilitasse de compreender e participar da pesquisa.

Quarenta idosos preencheram os critérios para a participação do estudo. Porém, três deles foram excluídos por terem participado do estudo piloto, realizado a fim de verificar a viabilidade do estudo e dos instrumentos. Dessa maneira, foram sujeitos dessa pesquisa 37 idosos, sendo 14 indivíduos do sexo feminino e 23 do sexo masculino.

A coleta de dados teve início após a leitura e assinatura do TCLE e foi realizada no período de setembro a dezembro de 
2007. Cada idoso foi entrevistado individualmente e a duração média de cada entrevista foi de 40 minutos.

Os instrumentos utilizados na coleta de dados foram: Questionário de Caracterização Sociodemográfica, Questionário de Avaliação do Sono e Questionário sobre Organização Cronológica das Rotinas Diárias. Todos os instrumentos foram elaborados previamente pelas pesquisadoras e estão fundamentados na literatura e em experiência anterior.

Para a caracterização sociodemográfica, o instrumento contemplou as seguintes variáveis: idade, sexo, estado civil, escolaridade, tempo de residência na ILPI, número de idosos com quem compartilhava o dormitório e atividades realizadas na instituição.

A qualidade do sono foi avaliada em duas etapas, por meio do Questionário de Avaliação do Sono. A primeira etapa consistiu em classificar a qualidade do sono de acordo com a presença dos seguintes critérios, compatíveis com sono de má qualidade: 1) latência para o início do sono igual ou superior a 30 minutos, 2) profundidade do sono reduzida, 3) dois ou mais despertares durante a noite, 4) ausência de sensação de bem estar ao acordar, 5) outras queixas espontâneas em relação ao sono. A ausência de queixas em quatro das cinco variáveis apresentadas foi interpretada como sono de boa qualidade. Na segunda etapa, o sono foi classificado de acordo com a avaliação do idoso, em resposta a uma única questão. Foi solicitado ao idoso que ele classificasse o próprio sono em positivo, negativo ou neutro. Em seguida, comparou-se a frequência dos critérios indicativos de sono de má qualidade com a classificação apontada pelo idoso.

O Questionário sobre Organização Cronológica das Rotinas Diárias apresentou as seguintes variáveis: horário de despertar, motivo de despertar, horário do banho, horário do almoço, horário de jantar, modo pelo qual reconhecia os horários das refeições, horário de dormir e outras atividades. A percepção da organização cronológica das rotinas foi considerada adequada quando os idosos responderam com coerência pelo menos quatro questões do instrumento.

Os dados foram analisados e descritos segundo as frequências absolutas e relativas.

\section{RESULTADOS}

A Tabela 1 traz as características sociodemográficas dos idosos institucionalizados participantes dessa pesquisa.

Os idosos eram, em sua maioria, do sexo masculino, inseridos na faixa etária de 70 a 79 anos (média de idade de 75,0 anos, desvio padrão $=7,0$ ), solteiros, com ensino primário completo e tempo de residência na ILPI entre 1,1 e 5,0 anos. Os dormitórios eram compartilhados, na maior parte das vezes, por até nove pessoas (Tabela 1).

Os dados sobre a organização cronológica em relação à rotina dos idosos são apresentados na Tabela 2.

A maioria dos idosos despertava entre seis e sete horas da manhã, por estarem habituados. Preferiam tomar banho de manhã, almoçavam às onze e meia, jantavam às cinco e meia da tarde e dormiam entre 19 e 22 horas. O toque do sino era o modo pelo qual os idosos reconheciam o horário das refeições (Tabela 2).
Tabela 1 - Características sociodemográficas dos idosos residentes na Instituição de Longa Permanência $(n=37)$. Campinas-SP, Brasil, 2007

\begin{tabular}{|c|c|c|c|}
\hline Variáveis & Categorias & $\mathbf{n}$ & $\%$ \\
\hline \multirow[t]{2}{*}{ Sexo } & Feminino & 14 & 38 \\
\hline & Masculino & 23 & 62 \\
\hline \multirow[t]{4}{*}{ Faixa etária } & 60 a 69 anos & 09 & 25 \\
\hline & 70 a 79 anos & 16 & 43 \\
\hline & 80 a 89 anos & 06 & 16 \\
\hline & Sem informação & 06 & 16 \\
\hline \multirow[t]{3}{*}{ Estado civil } & Solteiro & 19 & 52 \\
\hline & Separado & 03 & 08 \\
\hline & Viúvo & 15 & 40 \\
\hline \multirow[t]{3}{*}{ Escolaridade } & Analfabeto & 09 & 24 \\
\hline & Em alfabetização & 11 & 30 \\
\hline & Primário completo & 17 & 46 \\
\hline \multirow[t]{4}{*}{ Tempo de residência na ILPI } & 6 meses a 1,0 ano & 03 & 10 \\
\hline & 1,1 a 5,0 anos & 17 & 45 \\
\hline & 5,1 anos a 10,0 anos & 15 & 40 \\
\hline & Mais de 10 anos & 02 & 05 \\
\hline \multirow{3}{*}{$\begin{array}{l}\text { Número de pessoas que } \\
\text { compartilha o dormitório }\end{array}$} & 3 pessoas & 14 & 38 \\
\hline & 7 pessoas & 07 & 19 \\
\hline & 9 pessoas & 16 & 43 \\
\hline
\end{tabular}

Quinze idosos (40\%) realizavam atividades para a instituição, ou seja, auxiliavam em tarefas como carpir e pintar, cuidar dos animais, bater o sino que anuncia as refeições, ajudar na lavanderia. Atividades como "participar do baile aos domingos" (13\%), "ir à igreja pela manhã" (13\%), "frequentar a escola" (6\%), "frequentar a Terapia Ocupacional" (8\%), "realizar caminhadas" (13\%) e "fazer ginástica" (7\%) também foram mencionadas. Os horários preferidos para a realização das atividades foram "manhã" e "tarde".

Sete participantes referiram sono "pesado". Porém, apresentar sono "leve" foi a queixa da maioria dos idosos ( $n=30,81 \%)$ e os principais motivos relatados para tal ocorrência foram: ir ao banheiro à noite $(92 \%)$, ouvir o ronco dos companheiros de quarto $(16 \%)$, presença de pernilongos $(13 \%)$ e claridade $(5 \%)$.

Em relação aos idosos que despertavam para ir ao banheiro, quatro $(12 \%)$ o faziam quatro vezes por noite, 14 (41\%) três vezes, oito (24\%) duas vezes e outros oito (24\%) uma única vez.

Quanto ao cochilo, 21 idosos (57\%) referiram cochilar após o almoço e 16 idosos (43\%) relataram não cochilar, alegando que poderiam 'perder o sono' durante a noite. A duração do cochilo variou de dez a 180 minutos. Para sete idosos (33\%) o cochilo durava entre dez e 30 minutos, para oito idosos (38\%) entre 40 e 60 minutos e para seis idosos (29\%) a duração era superior a 60 minutos.

Verificou-se que 11 idosos (30\%) referiram iniciar o sono rapidamente, sem estimar o tempo em minutos; 12 (32\%) afirmaram demorar entre dez e 30 minutos para conciliar o sono; sete (19\%) relataram demorar entre 40 e 60 minutos e os sete restantes (19\%) disseram que esse intervalo variava entre 70 e 180 minutos. 
Tabela 2 - Organização cronológica em relação à rotina segundo a percepção dos idosos residentes na Instituição de Longa Permanência estudada $(n=37)$. Campinas-SP, Brasil, 2007

\begin{tabular}{|c|c|c|c|}
\hline Variáveis & Categorias & $\mathbf{n}$ & $\%$ \\
\hline \multirow[t]{3}{*}{ Horário de despertar } & $04 h-05 h$ & 17 & 46 \\
\hline & $06 h-07 h$ & 18 & 49 \\
\hline & $08 h-09 h$ & 02 & 05 \\
\hline \multirow[t]{5}{*}{ Motivo do despertar } & Enfermeira no quarto & 11 & 30 \\
\hline & Hábito & 15 & 40 \\
\hline & Relógio desperta & 06 & 16 \\
\hline & Barulho & 03 & 09 \\
\hline & Claridade & 02 & 05 \\
\hline \multirow[t]{4}{*}{ Horário do banho } & Manhã & 24 & 65 \\
\hline & Tarde & 07 & 19 \\
\hline & Noite & 04 & 11 \\
\hline & Sem preferência & 02 & 05 \\
\hline Horário de almoço & 11h30min & 37 & 100 \\
\hline \multirow{3}{*}{$\begin{array}{l}\text { Modo pelo qual reconhece } \\
\text { o horário do almoço }\end{array}$} & Toque do sino & 24 & 65 \\
\hline & Relógio de pulso & 11 & 30 \\
\hline & Fome & 02 & 05 \\
\hline Horário de jantar & 17h30min & 37 & 100 \\
\hline \multirow{3}{*}{$\begin{array}{l}\text { Modo pelo qual reconhece } \\
\text { o horário do jantar }\end{array}$} & Toque do sino & 26 & 70 \\
\hline & Relógio de pulso & 10 & 27 \\
\hline & Fome & 01 & 03 \\
\hline \multirow[t]{4}{*}{ Horário de dormir } & $19 h-20 h$ & 16 & 43 \\
\hline & $21 h-22 h$ & 16 & 43 \\
\hline & $23 h-24 h$ & 04 & 11 \\
\hline & Após as 24h & 01 & 03 \\
\hline
\end{tabular}

Tabela 3 - Frequência e proporção de queixas compatíveis com sono de má qualidade apresentadas pelos idosos residentes na Instituição de Longa Permanência ( $n=37)$. Campinas-SP, Brasil, 2007

\begin{tabular}{lll}
\hline Queixas & $\mathbf{n}$ & \% \\
\hline Latência do sono igual ou superior a 30 minutos & 19 & 51 \\
Profundidade do sono reduzida & 30 & 81 \\
Dois ou mais despertares noturnos & 26 & 70 \\
Bem estar ao despertar ausente & 04 & 11 \\
Outras queixas & 08 & 22 \\
\hline
\end{tabular}

A Tabela 3 mostra a frequência e proporção das queixas compatíveis com sono de má qualidade apresentadas pelos idosos.

Observou-se que sete idosos (19\%) não manifestaram queixas em quatro ou mais das cinco questões, ou seja, o sono foi considerado de boa qualidade de acordo com esses critérios. Para os outros 30 participantes (81\%), o sono foi classificado como de má qualidade.
Quanto à qualidade do sono classificada pelo próprio idoso, 18 deles (49\%) julgaram o sono como 'positivo', 11 (30\%) avaliaram-no como 'negativo' e oito (21\%), como 'neutro'.

\section{DISCUSSÃO}

O desafio do Brasil para este século é cuidar de uma população cada vez mais idosa com nível socioeconômico e educacional muitas vezes reduzido e com alta prevalência de condições crônicas de saúde ${ }^{(12)}$. Tradicionalmente, em nosso país, esse cuidado é realizado pelas famílias, conforme recomendação da Constituição Brasileira (artigo 230), Política Nacional do Idoso de $1994^{(13)}$ e Estatuto do Idoso aprovado em $2003^{(14)}$.

No entanto, as famílias têm sofrido mudanças na sua estrutura e na sua função. Atualmente, elas são menos extensas, com uma quantidade menor de filhos. Cresceu o número de divórcios e aumentou a participação das mulheres no mercado de trabalho, provocando uma alteração no papel social da mulher e nas relações de gênero tradicionais onde se considerava o homem como "provedor" e a mulher como "cuidado$\mathrm{ra}^{\prime \prime(15)}$. As mudanças atuais nos arranjos familiares têm influenciado a capacidade da família de cuidar de seus parentes mais frágeis, o que pode contribuir para uma carência assistencial dos idosos mais incapacitados ${ }^{(10)}$.

A demanda por novas modalidades de cuidados tende a crescer, em função desses novos arranjos familiares. O número de idosos brasileiros que deverão necessitar de cuidados prolongados poderá ascender de $30 \%$ a $50 \%$, dependendo dos avanços apresentados em relação às condições de autonomia dos idosos ${ }^{(10,16)}$. Estima-se que o Brasil, em 2020, terá o dobro de pessoas idosas sendo cuidadas por pessoas externas à família em relação ao ano de $2008^{(2)}$.

Em relação ao sexo, nesse estudo houve predomínio de homens institucionalizados. Esse dado vai de encontro a outros estudos encontrados na literatura, nos quais há predomínio de mulheres idosas institucionalizadas ${ }^{(10,17)}$. Em ILPIs, observa-se um predomínio de mulheres devido à feminização da velhice e, quando viúvas ou separadas, não se casarem novamente ${ }^{(10)}$. Sugere-se que a predominância masculina seja decorrente dos critérios de inclusão e exclusão adotados para a composição da amostra.

Os idosos jovens foram maioria nessa pesquisa. Essa predominância corrobora uma peculiaridade do recente envelhecimento no contexto nacional, o que se diferencia dos países desenvolvidos, nos quais predominam idosos mais velhos, isto é, de 80 anos e mais ${ }^{(18)}$.

Neste estudo, observa-se a predominância de mulheres e homens não casados. Esses resultados corroboram os achados do estudo sobre o apoio social dos idosos institucionalizados, realizado na Colômbia, no qual 48\% dos idosos institucionalizados eram solteiros, seguidos dos viúvos (30\%); apenas $11 \%$ dos idosos institucionalizados eram divorciados e $10 \%$ eram casados $^{(19)}$.

Quanto à escolaridade, 46\% dos entrevistados possuía o ensino primário completo. Na época em que esses idosos cresceram o nível de instrução formal não era apreciado. Aos meninos, cabia o cultivo da terra e às meninas, os afazeres 
domésticos. Além disso, muitos deles vivenciaram condições socioeconômicas precárias, dificultando o acesso à escola ${ }^{(20)}$.

O envelhecimento e a institucionalização acarretam modificações na qualidade de vida do idoso, da qual um dos componentes em evidência atualmente é a qualidade do sono. Autores apontam que os idosos comumente relatam cochilar, havendo um aumento da tendência ao sono no período da tarde ${ }^{(21)}$, o que é congruente com este estudo, no qual $57 \%$ dos idosos referiam cochilar após o almoço, com média de 63 minutos de duração.

O sono noturno insuficiente pode conduzir a um aumento da sonolência diurna em todos os grupos etários. Em idosos, a perturbação do sono noturno pode levar à privação de sono e ao prejuízo de sua capacidade de manter a vigília durante todo o dia. É difícil determinar a direção da relação causal entre o cochilo e a perturbação do sono noturno, a qual pode variar entre indivíduos. Alguns indivíduos podem dormir mais durante o dia para compensar uma privação do sono noturno, enquanto outros podem cochilar por outros motivos (por exemplo, inatividade) e, como resultado, desenvolvem dificuldades com o sono noturno(22). Em relação aos idosos, também têm sido implicados como contribuintes os frequentes despertares noturnos ${ }^{(23)}$.

O cochilo frequente, longo e não planejado pode ter impacto negativo sobre a qualidade do sono noturno e estar associado a consequências negativas para a saúde, tais como doença cardiovascular, aumento do risco de quedas e de comprometimento cognitivo ${ }^{(22)}$. Em contraste com estes efeitos negativos, curtos cochilos diurnos podem demonstrar efeitos positivos ao fornecer algum benefício em termos de vigilância aumentada. Embora esta questão tenha recebido relativamente pouca atenção na literatura de pesquisa, há algumas evidências de que cochilos possam ser benéficos se ocorrerem no momento adequado do dia e se tiverem uma duração adequada ${ }^{(24)}$.

Dentre os principais fatores que contribuem para o sono fragmentado e para os distúrbios do sono dos idosos institucionalizados encontram-se os despertares noturnos frequentes, que podem produzir sonolência diurna excessiva. Esses despertares podem ser gerados por fatores extrínsecos, tais como a entrada de funcionários no quarto para realizar cuidados e a agitação dos residentes com quem o quarto é compartilhado. Fatores intrínsecos são também causas de sono fragmentado e de distúrbios do sono, destacando-se as condições clínicas e psiquiátricas, prevalentes nas ILPIs ${ }^{(9)}$.

Entre os idosos deste estudo, 30\% classificaram a qualidade do sono como negativa, enquanto que, pelos critérios estabelecidos a partir das queixas relatadas, $81 \%$ dos idosos apresentavam sono de má qualidade. Observou-se, portanto, uma contradição que se mostra frequente nos estudos referentes à qualidade do sono dos idosos, ou seja, a discrepância entre a qualidade subjetiva e a qualidade aferida segundo critérios. Diversos fatores implicados na má qualidade do sono podem ser vistos pelo idoso como um padrão "normal" no envelhecimento, prejudicando a busca de auxílio ou de orientações a respeito ${ }^{(9)}$.

É importante destacar que os principais motivos de queixas referidas pelos idosos foram o sono de pouca profundidade ('leve'), as interrupções frequentes durante a noite devido à nictúria e a dificuldade em iniciar o sono (latência do sono), cuja média foi de 64 minutos. Apenas $11 \%$ de idosos referiram queixas ao despertar. A maior parte deles relatava aspectos positivos, como "acordar bem, descansada, alegre, feliz, animada".

Nota-se que a instituição apresenta horário pré-estabelecido para a realização de refeições, 11h30min e 17h30min, limitando a liberdade do idoso à rotina do local em que vivem. Nesse sentido, autores apontam que a qualidade da vigília influencia a qualidade do sono ${ }^{(6)}$.

Em relação ao banho, a maioria dos idosos da instituição pesquisada o realiza no período da manhã. Várias instituições preconizam o período da manhã para a higiene corporal e consideram o nível de dependência do idoso no momento de sua realização. Em geral, há um único banheiro no quarto, fazendo com que os idosos se organizem para tomar banho em diferentes horários e nem sempre o fazem de acordo com sua preferência.

Aqui só tem um banheiro por quarto, imagina os nove no banheiro ao mesmo tempo? Por isso vejo o horário do banheiro vazio. (Sujeito 34)

Quanto às medicações, cada idoso adapta-se geralmente aos horários estabelecidos pela instituição. Os responsáveis pela administração dos medicamentos são os membros da equipe de enfermagem, a qual inclui o enfermeiro, o técnico e o auxiliar de enfermagem. Um estudo sobre a humanização do cuidado aos idosos institucionalizados afirma que a vigilância à saúde do idoso deve ser contínua e ser realizada por profissionais específicos da geriatria/gerontologia, por meio da atuação dos profissionais de enfermagem ${ }^{(25)}$.

Dentre as queixas apontadas pelos idosos, destaca-se o fato dos profissionais de enfermagem entrarem no dormitório pela manhã ou pela noite, para o cuidado a um dos residentes, acendendo a luz e interrompendo o sono dos demais.

Já é difícil pra gente pegar no sono e quando pega, vem um enfermeiro e acende a luz para dar remédio ao colega do quarto... acordo e não durmo mais. (Sujeito 28)

É importante ressaltar que essas rotinas citadas anteriormente podem servir como marcações temporais para os idosos de uma ILPI. A literatura aponta que a organização cronológica bem estruturada de rotinas contribui para a boa qualidade do sono $^{(26)}$. Assim, neste estudo, acreditava-se que os idosos que demonstrassem boa percepção da organização cronológica de sua rotina na instituição apresentariam menor número de queixas em relação ao sono, ou seja, sono de melhor qualidade do que idosos que não conseguissem reconhecer a alocação cronológica dessas rotinas. Isso não se confirmou, pois $100 \%$ dos idosos demonstraram boa percepção das rotinas diárias segundo critérios estabelecidos, porém $81 \%$ relataram queixas compatíveis com má qualidade do sono. Esse achado leva à reflexão sobre a importância de serem realizadas atividades que tenham significado para o idoso. 
Diante dos achados do presente estudo, ressalta-se a importância do trabalho desenvolvido pelos profissionais de saúde nas ILPIs, as quais insurgem como uma alternativa não familiar para suprir as necessidades dessa população. O enfermeiro deve considerar as condições específicas de cada idoso, a fim de oferecer uma assistência gerontogeriátrica de qualidade em busca do bem estar desses indivíduos e do envelhecimento saudável(17).

\section{CONCLUSÕES}

Observou-se que os idosos institucionalizados percebem adequadamente a organização cronológica de suas rotinas, porém essa percepção parece não contribuir para a boa qualidade do sono, visto que a maioria deles apresentou queixas compatíveis com sono de má qualidade. Além disso, há uma contradição entre a qualidade do sono avaliada por critérios estabelecidos a partir das queixas, e a qualidade do sono classificada pelo idoso.
O conhecimento da qualidade do sono de idosos institucionalizados é importante para o desenvolvimento de estratégias capazes de assistir às demandas crescentes dessas pessoas, haja vista que o sono inadequado pode interferir na saúde física e cognitiva, além de afetar negativamente a qualidade de vida desses indivíduos. Assim, os enfermeiros devem intervir na promoção de saúde com ações que minimizem ou previnam esses problemas, a começar com a educação em saúde e avaliação detalhada e abrangente da qualidade do sono.

Além disso, atitudes dessa natureza implicam, para o enfermeiro, não somente em buscar um conhecimento de vanguarda como também em defender, a partir do mesmo, as pessoas sob seus cuidados. Uma vez identificada a importância do sono na manutenção e recuperação da saúde, torna-se um dever do profissional buscar os meios de promover o sono de boa qualidade para os seus pacientes e conscientizar os demais profissionais a esse respeito.

\section{REFERÊNCIAS}

1. Instituto Brasileiro de Geografia e Estatística [Internet]. [SI]: IBGE [acesso em 20 de setembro de 2011]. Sinopse do resultado do censo de 2010; [1 tela]. Disponível em: http://www.censo2010.ibge.gov.br/sinopse/webservice/

2. Banco Mundial. Envelhecendo em um Brasil mais veIho [Internet]. Washington: [s.n.]; 2011 [acesso em 04 de junho de 2014]. Disponível em: http://siteresources.worldbank.org/BRAZILINPOREXTN/Resources/3817166-1302102548192/Envelhecendo_Brasil_Sumario_Executivo.pdf

3. Oliveira BHD, Yassuda MS, Cupertino APFB, Neri AL. Relações entre padrão do sono, saúde percebida e variáveis socioeconômicas em uma amostra de idosos residentes na comunidade - Estudo PENSA. Ciênc Saúde Coletiva. 2010;15(3):851-60.

4. Martin J, Shochat T, Ancoli-Israel S. Assessment and treatment of sleep disturbances in older adults. Clin Psychol Rev. 2000;20(6):783-805.

5. Ancoli-Israel S, Moore PJ, Jones V. The relationship between fatigue and sleep in cancer patients: a review. Eur J Cancer Care. 200110(4):245-55.

6. Costa SV, Ceolim MF, Neri AL. Problemas de sono e suporte social: estudo multicêntrico - Fragilidade em idosos brasileiros. Rev Latinoam Enferm. 2011;19(4):920-7.

7. Sforza E, Gauthier M, Crawford-Achour E, Pichot V, Maudoux D, Barthélémy JC, et al. A 3- year longitudinal study of sleep disordered breathing in the elderly. Eur Respir J. 2012;40(3):665-72.

8. Shochat T, Loredo J, Ancoli-Israel S. Sleep disorders in the elderly. Curr Treat Options Neurol. 2001;3(1):19-36.

9. Araújo CLO, Ceolim MF. Qualidade do sono de idosos residentes em instituição de longa permanência. Rev Esc Enferm USP. 2010;44(3):619-26.
10. Camarano AA, Kanso S. As instituições de longa permanência para idosos no Brasil. Rev Bras Estud Popul. 2010;27(1):232-5.

11. Voyer P, Verreault R, Mengue PN, Morin CM. Prevalence of insomnia and its associated factors in elderly long-term care residents. Arch Gerontol Geriatr. 2006;42(1):1-20.

12. Ramos LR. Fatores determinantes do envelhecimento saudável em idosos residentes em centro urbano: Projeto Epidoso, São Paulo. Cad Saúde Pública. 2003;19(3):793-8.

13. Brasil. Lei no 8.842, de 04 de janeiro de 1994. Dispõe sobre a política nacional do idoso, cria o Conselho Nacional do Idoso e dá outras providências. Diário Oficial da União 05 jan 1994;Seção 1.

14. Brasil. Lei $n^{\circ} 10.741$, de 01 de outubro de 2003. Dispõe sobre o Estatuto do Idoso e dá outras providências. Diário Oficial da União 03 out 2003;Seção 1.

15. Walsh F. Normal family processes: growing diversity and complexity. 4. th ed. New York: Guilford; 2012.

16. Camarano AA, Kanso S. Perspectivas de crescimento para a população brasileira: velhos e novos resultados [Internet]. Rio de Janeiro (RJ): IPEA; 2009 [acesso em 04 de junho de 2014]. Disponível em: http://www.ipea.gov.br/ portal/images/stories/PDFs/TDs/td_1426.pdf

17. Oliveira PB, Tavares DMS. Condições de saúde de idosos residentes em Instituição de Longa Permanência segundo necessidades humanas básicas. Rev Bras Enferm. 2014;67(2):241-6.

18. Sousa AI, Silver LD. Perfil sociodemográfico e estado de saúde autorreferido entre idosas de uma localidade de baixa renda. Esc Anna Nery Rev Enferm. 2008;12(4):706-16.

19. Cardona-Arango D, Estrada-Restrepo A, Chavarriaga-Maya LM, Segura-Cardona AM, Ordoñez-Molina J, Osorio-Gomez JJ. Apoyo social dignificante del adulto mayor 
institucionalizado. Rev Salud Pública. 2010;12(3):414-24.

20. Del Duca GF, Silva SG, Thumé E, Santos IS, Hallal PC. Indicadores da institucionalização de idosos: estudo de casos e controles. Rev Saúde Pública. 2012;46(1):147-53.

21. Xu Q, Song Y, Hollenbeck A, Blair A, Schatzkin A, Chen $\mathrm{H}$. Day napping and short night sleeping are associated with higher risk of diabetes in older adults. Diabetes Care. 2010;33(1):78-83.

22. Ficca G, Axelsson J, Mollicone DJ, Muto V, Vitiello MV. Naps, cognition and performance. Sleep Med Rev. 2010;14(4):249-58.

23. Monk TH, Buysse DJ, Carrier J, Billy BD, Rose LR. Effects of afternoon "siesta" naps on sleep, alertness, performance, and circadian rhythms in the elderly. Sleep. 2001;24(6):680-7.

24. Martin JL, Ancoli-Israel S. Napping in older adults. Sleep Med Clin. 2006;1(2):177-86.

25. Fragoso V. Humanização dos cuidados a prestar ao idoso institucionalizado. Rev IGT [Internet]. 2008 [acesso em 04 de junho de 2014];5(8):51-61. Disponível em: file://C:/Users/Cliente/Downloads/IGTnR-2007-178.pdf

26. Geib LTC, Cataldo Neto A, Wainberg R, Nunes ML. Sono e envelhecimento. Rev Psiquiatr Rio Gd Sul. 2003;25(3):453-65. 